\title{
O sistema de saúde em Portugal: realizações e desafios
}

\author{
Health care system in Portugal: Accomplishments and Challenges
}

El sistema de salud en Portugal: realizaciones y desafios

\section{Paulino Artur Ferreira de Sousa ${ }^{1}$}

\section{INTRODUÇÃO}

Os sistemas de saúde e os determinantes da sua evolução são de uma grande complexidade. Os sistemas de saúde evoluem sob o jogo de pressões contínuas de interesses e pontos de vista distintos. Para a governação da saúde, o desafio centra-se em conseguir compatibilizar a promoção e a protecção da saúde das pessoas, com a necessidade de incentivar o crescimento económico da comunidade.

Os últimos 30 anos de políticas de saúde em Portugal produziram várias mudanças de rumo e de enfoque. Por vezes, tem-se mesmo a sensação de que as mudanças de política são demasiado rápidas para serem acompanhadas pelo sistema, e que este acaba por evoluir de forma independente e ao seu ritmo.

A evolução do sistema de saúde português tem sido marcada, nos últimos anos, por um conjunto de factores que poderão ser analisados em quatro planos distintos: a questão da responsabilidade social e individual no financiamento dos cuidados de saúde; a possibilidade de se evoluir para um Estado Garantia (que para além de regular, seja prestador de cuidados e exigente na saúde); a aposta na centralidade do cidadão, no contexto da sociedade do conhecimento e da inovação; e, por último, a gestão de mudança nos sistemas de saúde.

Este artigo apresenta uma análise da evolução do Sistema de Saúde Português nos últimos 30 anos, centrado na análise das Reformas da Saúde em Portugal, recorrendo à análise documental com recurso a relatórios da Organização Mundial de Saúde (OMS) e de diversos Observatórios de Sistemas de Saúde ${ }^{(1-3)}$.

\section{A SAÚDE EM PORTUGAL}

O grande desenvolvimento científico, tecnológico, social e económico verificado nas últimas décadas, permitiu resolver muitos dos problemas de saúde do passado, mas, contribuiu para que hoje sejamos confrontados com problemas novos e mais complexos, entre outros, as alterações nas necessidades em cuidados de saúde motivadas pelo aumento da esperança de vida, envelhecimento progressivo da população, maior incidência e prevalência de doenças crónicas, o que origina novos desafios aos sistemas de saúde. Este contexto gera novos problemas sociais e de saúde com elevada ênfase na acessibilidade a serviços de saúde, na incapacidade agravada com o isolamento (com a consequente dependência de estruturas de apoio), no aumento do número de famílias com recursos económicos escassos.

Conhecer a evolução do sistema de saúde português ajuda-nos a compreender melhor os factores que mais influem sobre o seu desenvolvimento e sobre a sua configuração actual. Comecemos então por conhecer primeiro alguns dados sobre a saúde em Portugal:

${ }^{1}$ Doutor em Enfermagem, Professor Coordenador da Escola Superior de Enfermagem do Porto, Unidade de Investigação da Escola Superior de Enfermagem do Porto - USIESEP - Porto - Portugal. 
Em primeiro lugar, alguns aspectos centrados em dados sócio-demográficos da população portuguesa ${ }^{(3)}$ :

Tabela 1 - Dados sócio-demográficos da população portuguesa (1970, 1980, 1990, 2000 e 2004)

\begin{tabular}{lrrrrr}
\hline & $\mathbf{1 9 7 0}$ & $\mathbf{1 9 8 0}$ & $\mathbf{1 9 9 0}$ & $\mathbf{2 0 0 0}$ & $\mathbf{2 0 0 4}$ \\
\hline Age dependency ratio (dependants to working-age population) & 0.61 & 0.57 & 0.51 & 0.48 & 0.49 \\
Birth rate, crude (per 1000 people) & 20.00 & 16.20 & 11.80 & 11.60 & 10.40 \\
Death rate, crude (per 1000 people) & 10.30 & 9.70 & 10.40 & 10.50 & 9.70 \\
Fertility rate, total (births per woman) & 2.76 & 2.19 & 1.43 & 1.52 & 1.42 \\
Population ages (-14 (\% of total) & 28.80 & 25.90 & 20.40 & 16.20 & 15.90 \\
Population ages 15-64 (\% of total) & 62.00 & 63.60 & 66.20 & 67.60 & 67.20 \\
Population ages 65 and above $\%$ of total) & 9.20 & 10.50 & 13.40 & 16.10 & 16.90 \\
Population density (people per Km $\left.{ }^{2}\right)$ & 98.80 & 107.00 & 108.00 & 112.00 & 115.00 \\
Population growth (annual \%) & -0.58 & 1.08 & -0.41 & 0.51 & 0.58 \\
Population, female (\% of total) & 52.60 & 51.60 & 51.80 & 51.80 & 51.70 \\
Population, total (thousand) & 9040 & 9770 & 9900 & 10200 & 10500 \\
Urban population (\% of total) & 25.90 & 29.40 & 46.70 & 53.00 & 55.10 \\
\hline Sorat
\end{tabular}

Source: World Bank, 2006

Como podemos constatar através da Tabela 1, em 1970 apenas 25,9\% da população vivia em áreas urbanas, tendo este valor aumentado para 29,4\% em 1980, 46,7\% em 1990,53,0\% em 2000, e 55,1\% até 2004, segundo os dados disponíveis. O rácio de dependência caiu de 0,57 em 1980 para 0,49 em 2004 (com base na relação das pessoas com 15 e mais de 65 anos de idade para o restante da população). A idade média da população tem vindo a aumentar. Outro dado relevante prende-se com o número de nascimentos, que tem vindo a decrescer continuamente desde 1970 (20,0 vivo nascimentos por 1000 habitantes) e, em 1990, a taxa bruta de natalidade em Portugal foi 11,80 nascidos vivos por 1000 habitantes. Em 2004, o número de nascimentos por 1000 habitantes tinha diminuído para 10,4. As recentes projecções mostram que a população portuguesa ainda pode mostrar um ligeiro aumento durante a próxima década, mas irá diminuir a partir de 2010. É evidente que o aumento da proporção de pessoas com mais de 65 anos e a diminuição da população de 15 anos de idade irá resultar num efeito de "duplo envelhecimento", pelo que o cenário destas estimativas parece confirmar que em Portugal uma diminuição da população é quase inevitável.

Centremos agora a nossa atenção em alguns em indicadores de saúde e de mortalidade da população portuguesa ${ }^{(3)}$ :

Em 2005, a esperança média de vida à nascença em Portugal foi de 78,2 anos, verificando-se uma diferença entre as estimativas da esperança de vida para os homens e as mulheres: em 2005 os valores foram 81,4 anos para as mulheres e 74,9 anos para os homens. De acordo com a Tabela 2, a expectativa de vida ao nascer tem vindo a melhorar. Esta tendência tem continuado a desenvolver a partir de meados dos 1980, fazendo a esperança de vida em Portugal convergir com a média da União Europeia (UE).

A taxa de mortalidade infantil diminuiu significativamente entre 1970 e 1990, e diminuiu de 10,8 por 1.000 em 1991 para 3,5 por 1000, em 2005, abaixo da média da mortalidade infantil para 15 Estados-Membros (4,1 por 1000 nascidos vivos em 2004).

Tabela 2 - Indicadores de saúde e de mortalidade da população portuguesa (1980, 1990, 2000, 2004 e 2005)

\begin{tabular}{lrrrrr}
\hline & $\mathbf{1 9 8 0}$ & $\mathbf{1 9 9 0}$ & $\mathbf{2 0 0 0}$ & $\mathbf{2 0 0 4}$ & $\mathbf{2 0 0 5}$ \\
\hline Life expectancy at birth, female (years) & 74.6 & 77.6 & 80.3 & 81.6 & 81.4 \\
Life expectancy at birth, male (years) & 67.5 & 70.6 & 73.2 & 74.9 & 74.9 \\
Life expectancy at birth, Total (years) & 71.2 & 74.1 & 76.8 & 78.3 & 78.2 \\
Mortality rate (per 1000 female adults) & 8.7 & 9.6 & 9.5 & 9.0 & - \\
Mortality rate (per 1000 male adults) & 10.6 & 11.1 & 11.2 & 10.5 & - \\
Mortality rate, crude (per 1000) & - & - & - & - & 10.2 \\
Infant deaths per 1000 live births & 24.3 & 11.0 & 5.5 & 3.9 & 3.5 \\
Probability of dying before age 5 years (per 1000 live births) & 29.2 & 14.0 & 7.3 & 5.2 & - \\
Souces: WHO Regional Office for Europe, 2007; OECD, 2006c; INE, 2005a (for all 2005 data)
\end{tabular}

É claro que este avanço significativo nos indicadores de Saúde Pública, como a mortalidade infantil, não serve apenas aos serviços de saúde. O desenvolvimento da rede de abastecimento de água potável a todo o País e o saneamento básico, fruto de mudanças após 1980, terão contribuído drasticamente para reduzir a mortalidade infantil.

Neste contexto, as alterações demográficas verificadas em Portugal, à semelhança do ocorrido em toda as sociedades sem 
excepção, que se traduzem pela inversão das pirâmides etárias, vieram colocar desafios para os quais não estávamos preparados. Segundo o Departamento de Estatística da União Europeia (Eurostat), Portugal em 2050 será o quarto país da UE com maior percentagem de idosos (31,9\%), só ultrapassado por Espanha (35,6\%), Itália (35,3\%) e Grécia (32,5\%). Esta realidade que se espelha nas sociedades industrializadas e que ganhou um impacto social relevante é um efeito cumulativo da diminuição da mortalidade e da natalidade e do aumento da esperança média de vida, consequência directa dos progressos da medicina e das transformações económicas e sociais. Não temos dúvidas que estas situações determinam novas necessidades em saúde, por isso, urge organizar respostas adequadas à crescente necessidade de cuidados continuados, de forma personalizada, de qualidade e em proximidade, muito diferente do modelo de intervenção anteriormente utilizado ${ }^{(2)}$.

\section{A EVOLUÇÃo do SISTEMA de SAÚde PORTUGUÊS}

Durante os últimos 30 anos do século passado, Portugal sofreu sucessivamente, em períodos de tempo particularmente curtos, transformações de grande importância: democratização e descolonização (1974), entrada na Comunidade Economica Europeia (CEE) (1985) e integração na União Monetária Europeia (2000), num ambiente de rápida transição de paradigma tecnológico, nomeadamente na área da saúde. Podemos então afirmar que, em Portugal, as políticas de saúde evoluíram significativamente nos últimos 30-35 anos, às quais podemos associar períodos distintos, que correspondem, de certa forma, a diferentes "agendas políticas":

- antes dos anos 1970 (sistema de saúde muito fragmentado);

- do início dos anos 1970 a 1985 (estabelecimento e expansão do Serviço Nacional de Saúde);

- de 1985 a 1995 (Período de expansão do Serviço Nacional de Saúde numa rede mais ampla de prestadores de cuidados do sector social e privado, de regionalização e coordenação do Serviço Nacional de Saúde, de incremento da participação do sector privado, de construção hospitalar e de tentativa de separação do exercício médico entre sectores privado e público);

- de 1995 a 2002 (período marcado por uma tentativa de estimular formas diferentes de gestão e organização empresarialização pública de instituições de saúde -, e pela adopção de uma estratégia de saúde explícita);

- de 2002 até ao momento actual (expansão e reorientação das políticas definidas anteriormente, com uma nova filosofia em relação aos papéis dos sectores público, privado e social).

\section{O sistema de saúde português antes de 1970}

Em Portugal, a evolução do sistema de saúde desde os anos 30 foi marcada pela ideologia corporativa que imprimiu em toda a legislação uma concepção de assistência médico-sanitária, predominantemente caritativa. A filosofia subjacente preconizava a não intervenção do Estado nos problemas de saúde; o seu papel era meramente supletivo às iniciativas particulares do indivíduo ou de outras instituições nomeadamente da Previdência Social.

Após a segunda guerra mundial e até início dos anos 60, o hospital foi considerado o elemento central do sistema de saúde, ao que se associou a ideia da "Regionalização hospitalar": hospitais agregados em três níveis: por conselho, por distrito e por zonas (hospitais centrais), congregando vários distritos, cooperando entre si e ampliando as suas responsabilidades no sentido ascendente. É de realçar que nesta época o papel assumido pelas Misericórdias, que possuíam a maioria dos hospitais, foi um papel importante na coordenação entre as actividades de assistência social e cuidados de saúde.

Nos anos 60 procurou-se a integração de todas as actividades de saúde, passando o hospital a ser considerado uma estrutura superior, mas não central do sistema.

No inicio dos anos 70, Portugal apresentava indicadores socioeconómicos e de saúde muito desfavoráveis no contexto da Europa Ocidental de então, a título de exemplo: uma taxa de mortalidade infantil de 58.6 (5.0 em 2001), $37 \%$ de partos hospitalares (99\% em 2001).

O sistema de saúde português estava muito fragmentado: alguns grandes hospitais do Estado, uma extensa rede de hospitais das Misericórdias, postos médicos dos Serviços Médico Sociais da Previdência; Serviços de Saúde Pública (centros de saúde a partir de 1971); médicos municipais; sector privado especialmente desenvolvido na área do ambulatório. De salientar que a capacidade de financiar os serviços públicos da saúde era muito limitada - a despesa com a saúde era de $2,8 \%$ do PIB, em 1970.

O sistema de saúde português (1971-1985): estabelecimento e expansão do Serviço Nacional de Saúde

A reforma do sistema de saúde e da assistência, legislada em 1971 (conhecida como "a reforma de Gonçalves Ferreira"), que incluiu o estabelecimento dos "Centos de Saúde", foi concebida no espírito daquilo que, sete anos mais tarde, vem a ser declarado, em Alma Ata, como "cuidados de saúde primários". Apesar de uma implementação limitada, forneceu a base para o futuro Serviço Nacional de Saúde. A revolução democrática do 25 de Abril de 1974 
e a Constituição de 1976 mudaram Portugal profundamente: novas políticas sociais; intervenção clara do Estado na definição da política de saúde, no planeamento e na execução; e, intervenção face às assimetrias regionais e sociais, com o reconhecimento do direito à saúde por parte de todos os cidadãos.

O Estado assume-se como responsável pela concretização do direito à protecção da saúde previsto na Constituição, o que culmina com a publicação em 1979 da Lei do Serviço Nacional de Saúde ${ }^{(3)}$. A criação de um Serviço Nacional de Saúde (SNS) foi vista como a resposta mais adequada à necessidade de uma cobertura, mais extensa e equitativa, de serviços de saúde.

A nova constituição estabelecia que "todos têm direito à protecção da saúde" que se realizaria "pela criação de um serviço nacional de saúde universal, geral e gratuito".

A Lei do Serviço Nacional de Saúde (1979) declara que o acesso ao SNS deve ser garantido a todos os cidadãos independentemente da sua condição social ou económica. Facto que com a revisão da Constituição Portuguesa em 1989, procede à mudança de "serviço nacional de saúde" "gratuito" para "tendencialmente gratuito".

Os anos 70 não foram tempos fáceis para a reforma. Para um país que experimentava o início da democracia e o processo da descolonização, as exigências de gestão do SNS foram um enorme desafio.

Após uma década de implementação dos centros de saúde (centros de saúde de $1^{a}$ geração), centrados no desenvolvimento de actividades predominantes na área da saúde pública, perdeu-se a oportunidade de lhes fornecer o desenvolvimento organizacional necessário para melhorar a acessibilidade. Este facto levou à sua associação com os Serviços Médico-Sociais da Previdência, que desenvolviam actividades essencialmente curativas, dando origem aos centros de saúde de $2^{a}$ geração (1984). Foi uma época onde se procurou recuperar o atraso em termos quantitativos, mas onde se verificaram acentuadas lacunas em matéria da qualidade de cuidados de saúde.

\section{O sistema de saúde português (1985-1995): regionalização do SNS e novo papel para o sector privado}

Esta década foi marcada por uma estabilidade política sem precedentes desde a revolução de Abril. Durante este período (em 1986), Portugal tornou-se membro da CEE (agora União Europeia). Esta adesão permitiu o acesso ao financiamento europeu para desenvolvimento de infra-estruturas sociais e económicas, onde se incluía a área da saúde. Torna-se, por isso, possível a expansão de instalações e equipamentos do SNS, o que assegura que uma proporção crescente da riqueza do país fosse destinada à saúde.

Um dos pontos centrais na Reforma da Saúde em Portugal registou-se em 1990, com a publicação da Lei de Bases da Saúde ${ }^{(4)}$, que define o sistema de saúde português como uma estrutura que visa a efectivação do direito à protecção da saúde. O Estado passa a actuar através de serviços próprios, celebrando acordos com entidades privadas para a prestação de cuidados e apoia e fiscaliza a restante actividade privada na área da saúde. Neste sentido, os cidadãos e as entidades, públicas e privadas, devem colaborar na criação de condições que permitam o exercício do direito à protecção da saúde e a adopção de estilos de vida saudáveis. A saúde passa a ser da responsabilidade não só do Estado mas também de cada indivíduo, e das iniciativas sociais e privadas

Com a publicação da Lei de Bases da Saúde e do Estatuto do SNS ${ }^{(5)}$ (definem-se as entidades intervenientes no sistema de saúde). O sistema de saúde português passa a ser caracterizado pela coexistência de três sistemas articulados entre si: o Serviço Nacional de Saúde (abrangendo todas as instituições e serviços oficiais prestadores de cuidados de saúde dependentes do Ministério da Saúde); subsistemas de saúde públicos de apoio (entidades públicas que desenvolvem actividades de promoção, prevenção e tratamento na área da saúde); e, todas as entidades privadas e todos os profissionais livres que acordem com o SNS a prestação de cuidados ou de actividade de saúde.

Tornou-se clara a necessidade de alterações de gestão e organizacionais para melhorar a efectividade e eficiência do sector da saúde, das quais destacámos:

- Um novo papel para o sector privado - procura-se estimular a intervenção do sector privado na área da saúde, incluindo a gestão privada de instituições de saúde.

- A regionalização e integração do SNS - Em 1993 foram estabelecidas cinco regiões administrativas de saúde (Administrações Regionais de Saúde) assim como "unidades funcionais" entre hospitais e centros de saúde. Estas últimas tinham como objectivo conseguir uma melhor integração entre cuidados primários, secundários e terciários.

- A introdução de taxas moderadoras - em 1990, o Governo introduziu taxas moderadoras no SNS no acesso a determinados cuidados de saúde, com excepção para grupos de risco e economicamente desfavorecidos.

\section{O sistema de saúde português (1995-2001): a abordagem segundo a "nova gestão pública" para a reforma do SNS}

Este período foi marcado por uma tentativa de estimular formas diferentes de gestão e organização.

Após dez anos de considerável estabilidade política - um governo com maioria parlamentar em oito destes dez anos - iniciou-se um novo ciclo político no final de 1995. Nos seis anos seguintes, o país é, novamente, governado 
por um governo de um só partido. No entanto, ao contrário do que aconteceu no ciclo político prévio, estes governos foram suportados por uma minoria parlamentar. No Parlamento, o suporte ao Governo era negociado caso a caso. Este ambiente político não era muito propício a grandes reformas. Neste contexto foi adoptado um processo de reforma cauteloso centrado em princípios da "nova gestão pública - new public management" aplicada à reforma do SNS, dos quais se destacam:

- A definição de uma estratégia de saúde:

De 1996 a 1999 foi desenvolvida uma ampla "Estratégia para o Virar do Século", que incluía metas de cinco e dez anos para ganhos em saúde e desenvolvimento de serviços.

- Empresarialização pública no desenvolvimento dos hospitais e centros de saúde. Foram realizadas durante este período algumas experiências de novas formas de gestão, mais flexível e autónoma, de "empresas públicas". Assiste-se ao início de uma série de projectos experimentais na reorganização dos cuidados de saúde primários. Estas experiências foram avaliadas positivamente e estimularam a adopção de sistemas de remuneração associada ao desempenho, numa base experimental, novas práticas de contratualização, critérios de qualidade e infra-estruturas de informação.

- As "agências de contratualização". Em 1996, as Administrações Regionais de Saúde iniciaram um processo que levou ao estabelecimento das "Agências de contratualização", que se destinavam a desenvolver perícia na análise, negociação e decisão da distribuição de recursos financeiros pelos serviços de saúde. Em 1999 foi aprovada legislação(6) para suportar a melhoria de coordenação das actividades de saúde ao nível local (sistemas locais de saúde), contudo, esta legislação não foi implementada.

Como verificámos, desde os anos 70, pode ser claramente identificado um esforço sustentado para melhorar a saúde e os serviços de saúde. Em larga medida, estes esforços consistiram no aumento do financiamento da saúde, na expansão dos serviços de saúde - quer em termos de instalações quer na adopção de novas tecnologias médicas e de informação - melhoria do acesso a medicamentos e esforços contínuos de melhorar a organização e gestão do SNS. No entanto, reformas bem-intencionadas foram muito frequentemente implementadas de forma incompleta, quer por limitações de gestão, resistência à mudança ou descontinuidade política. De facto, ao longo deste período de tempo, foi frequentemente observado que, no mesmo ciclo político, com o mesmo primeiro-ministro, a mudança de equipas ministeriais levou a alterações substanciais nas agendas políticas.

O sistema de saúde português após 2002 (expansão e reorientação das políticas definidas anteriormente, com uma nova filosofia em relação aos papéis dos sectores público, privado e social):

\section{O enfoque da governação do sistema de saúde: o financiamento em saúde}

O sistema de saúde português, tal como os seus congéneres europeus, tem-se defrontado com problemas de eventual insustentabilidade financeira. Ou seja, com a possibilidade de, a prazo, não ser financeiramente suportável, a menos que sejam introduzidas medidas no seu funcionamento que conduzam ao abrandamento do ritmo de crescimento da despesa pública com a saúde. Garantir a sustentabilidade financeira do sistema de saúde é uma condição indispensável para a defesa do modelo social, solidário e universal, que se pretende.

A questão da eficiência e eficácia do SNS, mais do que uma questão meramente orçamental, ou até apenas económica, é uma questão ética, ou seja, se o SNS não for eficiente, jamais poderá ser justo e flexível.

A evolução comparativa das situações dos países da União Europeia a 15 (UE15), relativamente à despesa total em saúde, à despesa pública e à relação entre as componentes pública e privada, permite-nos constatar que:

- A despesa total em saúde (Tabela 3) em percentagem do PIB tem vindo a crescer nos últimos anos em Portugal, à semelhança aliás do que tem acontecido noutros países da UE15. No entanto, desde 1995, o peso deste valor no PIB em Portugal, tem-se mantido acima da média dos países da UE15. Em 2004, os gastos chegaram a 10\% do PIB.

Se atendermos, por exemplo à dimensão da despesa pública, verificamos que:

- A despesa pública em saúde em percentagem do Produto Interno Bruto (PIB) tem vindo a crescer nos últimos anos em Portugal, à semelhança aliás do que tem acontecido noutros países da UE15 (mas com menor ênfase). Em 2004, os gastos públicos em saúde chegaram aos 7,4\% do PIB, 0,7\% acima da média dos países da UE15, (Tabela 4).

Se analisarmos a relação Público/Privado na Despesa em Saúde verificámos (através da Figura 1) que Portugal tem uma participação pública em saúde ligeiramente abaixo da média da UE a 15 países. Ou seja, gastamos mais do que a média da UE15 relativamente à riqueza produzida no país. No entanto, quando analisamos a despesa per capita, constatamos que somos os que gastamos menos, reflexo de sermos um país menos rico. Porém, quando se analisa a repartição dessa despesa entre o cidadão e o Estado (papel social), verificamos que somos dos países em que os cidadãos contribuem mais do que a média da UE15.

A análise da "Responsabilidade social e individual no financiamento da saúde", tendo em consideração os princípios básicos do "Estado do Bem-Estar", consagrados na ordem constitucional portuguesa: o princípio da solidariedade 
(redistribuição), ou seja, pagar de acordo com o rendimento para receber de acordo com as necessidades; o princípio da previdência, que se traduz por ir pagando no decurso da vida, enquanto se está bem, para receber quando se está doente, mais idoso e frágil; permite-nos constatar que esta fórmula respondeu, com assinalável êxito, no decurso do último século, principalmente na Europa, aos desafios do financiamento da saúde.

Tabela 3 - Despesa Total em Saúde (\% do PIB) (OCDE Health Data, 2006) ${ }^{(7)}$

\begin{tabular}{|c|c|c|c|c|c|c|c|c|c|c|c|c|c|c|c|c|}
\hline & 1970 & 1975 & 1980 & 1985 & 1990 & 1995 & 2000 & & 2001 & & 2002 & & 2003 & & 2004 & \\
\hline Alemanha & 6.2 & 8.6 & 8.7 & 9.0 & 8.5 & 10.1 & 10.3 & & 10.4 & & 10.6 & & 10.8 & & 10.6 & \\
\hline Ástria & 5.2 & 7.0 & 7.5 & 6.5 & 7.0 & 9.7 & 9.4 & & 9.5 & & 9.5 & & 9.6 & & 9.6 & \\
\hline Bélgica & 3.9 & 5.6 & 6.3 & 7.0 & 7.2 & 8.2 & 8.6 & & 8.7 & & 8.9 & & 10.1 & & n.d. & \\
\hline Dinamarca & n.d. & 8.7 & 8.9 & 8.5 & 8.3 & 8.1 & 8.3 & & 8.6 & & 8.8 & & 8.9 & & 8.9 & $\mathrm{e}$ \\
\hline Espanha & 3.5 & 4.6 & 5.3 & 5.4 & 6.5 & 7.4 & 7.2 & & 7.2 & & 7.3 & & 7.9 & & 8.1 & $\mathrm{e}$ \\
\hline Filândia & 5.6 & 6.2 & 6.3 & 7.1 & 7.8 & 7.4 & 6.7 & & 6.9 & & 7.2 & & 7.4 & & 7.5 & \\
\hline França & 5.3 & 6.4 & 7.0 & 7.9 & 8.4 & 9.4 & 9.2 & & 9.3 & & 10.0 & & 10.4 & & 10.5 & e \\
\hline Grécia & 6.1 & n.d. & 6.6 & n.d. & 7.4 & 9.6 & 9.9 & e & 10.4 & e & 10.3 & e & 10.5 & e & 10.0 & e \\
\hline Holanda & n.d. & 6.9 & 7.2 & 7.1 & 7.7 & 8.1 & 7.9 & & 8.3 & & 8.9 & & 9.1 & e & 9.2 & e \\
\hline Irlanda & 5.1 & 7.3 & 8.3 & 7.5 & 6.1 & 6.7 & 6.3 & & 6.8 & & 7.2 & & 7.2 & & 7.1 & \\
\hline Itália & n.d. & n.d. & n.d. & n.d. & 7.7 & 7.1 & 8.1 & & 8.2 & & 8.3 & & 8.4 & & 8.7 & \\
\hline Luxemburgo & 3.1 & 4.3 & 5.2 & 5.2 & 5.4 & 5.6 & 5.8 & & 6.4 & & 6.8 & & 7.7 & & 8.0 & e \\
\hline Portugal & 2.6 & 5.4 & 5.6 & 6.0 & 6.2 & 8.2 & 9.4 & & 9.3 & & 9.5 & & 9.8 & $\mathrm{e}$ & 10.1 & e \\
\hline Reino Unido & 4.5 & 5.5 & 5.6 & 5.9 & 6.0 & 7.0 & 7.3 & & 7.5 & & 7.7 & & 7.8 & & 8.1 & d \\
\hline Suécia & 6.8 & 7.6 & 9.0 & 8.6 & 8.3 & 8.1 & 8.4 & & 8.7 & & 9.1 & & 9.3 & & 9.1 & $\mathrm{e}$ \\
\hline UE15 & 4.8 & 6.5 & 7.0 & 7.1 & 7.2 & 8.0 & 8.2 & & 8.4 & & 8.7 & & 9.0 & & 9.0 & \\
\hline
\end{tabular}

Fonte: OCDE Health Data 2006, Outubro 2006

Legenda: n.d.-não disponível; d-Diferença de metodologia; e-estimativa

Tabela 4 - Despesa Pública em Saúde (\% do PIB) (OCDE Health Data, 2006) ${ }^{(8)}$

\begin{tabular}{|c|c|c|c|c|c|c|c|c|c|c|c|c|c|c|c|c|}
\hline & 1970 & 1975 & 1980 & 1985 & 1990 & 1995 & 2000 & & 2001 & & 2002 & & 2003 & & 2004 & \\
\hline Alemanha & 4.5 & 6.8 & 6.8 & 7.0 & 6.5 & 8.2 & 8.2 & & 8.3 & & 8.4 & & 8.5 & & 8.1 & \\
\hline Ástria & 3.3 & 4.8 & 5.1 & 4.9 & 5.1 & 6.7 & 6.6 & & 6.6 & & 6.7 & & 6.8 & & 6.8 & \\
\hline Bélgica & n.d. & n.d. & n.d. & n.d. & n.d. & 6.4 & 6.5 & & 6.6 & & 6.7 & & 7.2 & & n.d. & \\
\hline Dinam arca & n.d. & 7.5 & 7.9 & 7.3 & 6.9 & 6.7 & 6.8 & & 7.1 & & 7.3 & & n.d. & & n.d. & \\
\hline Espanha & 2.3 & 3.6 & 4.2 & 4.3 & 5.1 & 5.4 & 5.2 & & 5.2 & & 5.2 & & 5.5 & & 5.7 & $\mathrm{e}$ \\
\hline Filândia & 4.1 & 4.9 & 5.0 & 5.6 & 6.3 & 5.6 & 5.0 & & 5.2 & & 5.5 & & 5.7 & & 5.7 & \\
\hline França & 4.0 & 5.0 & 5.6 & 6.2 & 6.4 & 7.2 & 7.0 & & 7.1 & & 7.8 & & 8.2 & & 8.3 & e \\
\hline Grécia & 2.6 & n.d. & 3.7 & n.d. & 4.0 & 5.0 & 5.2 & $\mathrm{e}$ & 5.8 & $\mathrm{e}$ & 5.6 & $\mathrm{e}$ & 5.6 & $\mathrm{e}$ & 5.3 & $\mathrm{e}$ \\
\hline Holanda & n.d. & 4.7 & 5.0 & 5.0 & 5.2 & 5.7 & 5.0 & & 5.2 & & 5.5 & & 5.8 & e & 5.7 & e \\
\hline Irlanda & 4.1 & 5.8 & 6.8 & 5.7 & 4.4 & 4.8 & 4.6 & & 5.1 & & 5.4 & & 5.6 & & 5.7 & \\
\hline Itália & n.d. & n.d. & n.d. & n.d. & 6.1 & 5.1 & 5.8 & & 6.1 & & 6.2 & & 6.2 & & 6.5 & \\
\hline Luxembu rgo & 2.8 & 4.0 & 4.8 & 4.6 & 5.0 & 5.1 & 5.2 & & 5.6 & & 6.1 & & 7.0 & & 7.3 & $\mathrm{e}$ \\
\hline Portugal & 1.6 & 3.2 & 3.6 & 3.3 & 4.1 & 5.1 & 6.8 & & 6.7 & & 6.9 & & 7.2 & e & 7.4 & $\mathrm{e}$ \\
\hline Reino Unido & 3.9 & 5.0 & 5.0 & 5.0 & 5.0 & 5.8 & 5.9 & & 6.2 & & 6.4 & & 6.7 & & 7.0 & d \\
\hline Suécia & 5.9 & 6.8 & 8.3 & 7.8 & 7.5 & 7.0 & 7.1 & & 7.4 & & 7.8 & & 7.9 & & 7.7 & e \\
\hline UE15 & 3.6 & 5.2 & 5.5 & 5.6 & 5.5 & 6.0 & 6.1 & & 6.3 & & 6.5 & & 6.7 & & 6.7 & \\
\hline
\end{tabular}

Fonte: OCDE Health Data 2006, Outubro 2006

Legenda: n.d.-não disponível; d-Diferença de metodologia; e-estimativa

Em Portugal, tem-se vindo a incluir (e cada vez mais) medidas de responsabilidade individual no financiamento directo da saúde isto é, de "taxas moderadoras" de utilização dos serviços de saúde (consultas, internamentos, cirurgias de ambulatório, ...). Para alguns a inclusão destas medidas, sobre a forma de pagamentos no acto da utilização dos serviços, é desde já justificável, confiando que isto pode ser feito actualmente, protegendo satisfatoriamente os interesses dos utilizadores dos serviços de saúde; para outros, nas actuais circunstâncias do funcionamento do sistema de saúde português, este tipo de medidas acabarão por constituir uma barreira arriscada à utilização dos serviços de saúde por parte de quem precisa, acabando por "castigar" (e por vezes culpabilizar) pessoas por estarem doentes, no pior momento possível. É verdade que o mundo mudou substancialmente desde a adopção dos princípios da solidariedade e da previdência, e é portanto legítimo procurar encontrar novas formas de assegurar a sustentabilidade dos sistemas de protecção social, sem pôr em causa os seus princípios fundamentais.

Para o Observatório Português dos Sistemas de Saúde (OPSS) $)^{(1)}$, o SNS português, como o de outros países, tem-se desenvolvido na lógica da organização e financiamento da oferta de serviços de saúde à população do país, definindo alternativas ao desenvolvimento do SNS.

Olhar para um sistema de saúde quase exclusivamente pela organização e financiamento da oferta (Figura 2), constitui uma abordagem com uma forte base social de apoio no sistema político e nas profissões da saúde. $\mathrm{Na}$ 
opinião do relatório da OPSS, esta aproximação não permite fazer face adequadamente aos importantes desafios de acesso, qualidade, efectividade e eficiência que os sistemas de saúde têm que enfrentar de uma forma cada vez mais crítica. Na realidade, em Portugal, no decurso destes últimos anos, tem-se procurado deslocar o enfoque da governação do sistema de saúde dos "recursos" para os "resultados", da "organização e financiamento da oferta" para "melhoria do desempenho da oferta", tendo como principal instrumento desta transição, os processos de contratualização do desempenho. É evidente, que ao contrário da agenda da "oferta de recursos", a abordagem da "contratualização do desempenho" tem uma base social de apoio necessariamente mais ténue, o que justificará, em certa medida o ainda incipiente grau de desenvolvimento dos processos de contratualização no decurso da última década.

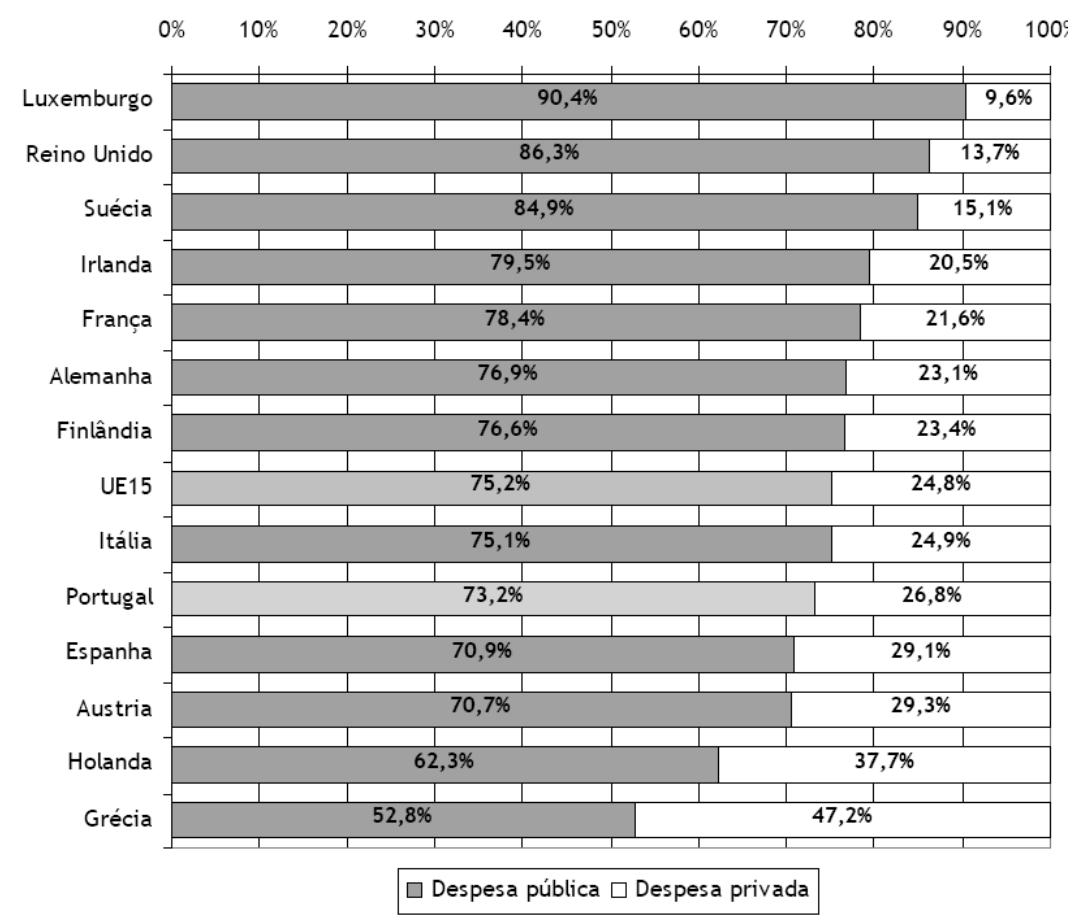

Inte: OCDE Health Data 2006, Outubro 2006

Figura 1- Relação Público / Privado na Despesa em Saúde (OCDE Health Data, 2006) ${ }^{(8)}$

- Olhar para um sistema de saúde pela lógica de "oferta de recursos", para uma de "procura de resultados" (Figura 3), o que implica a necessidade de termos cidadãos com escolhas informadas por resultados; processos de desenvolvimentos da qualidade dos cuidados baseados em resultados; metas de saúde e gestão de organizações de saúde centradas em resultados; contratualização do desempenho informada por resultados.

Temos assistido ao longo dos últimos anos, a um claro esforço para melhorar a saúde e os serviços de saúde disponibilizados. Entre outras medidas, um aumento significativo do financiamento da saúde, uma aposta na expansão

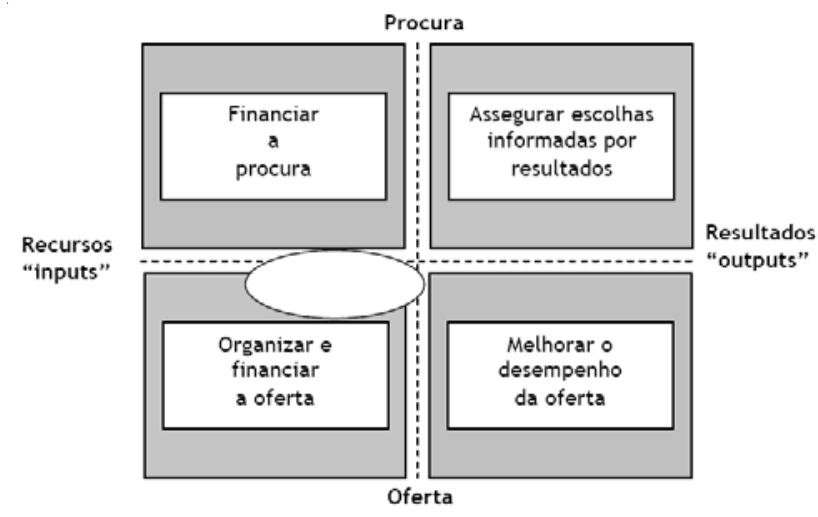

Figura 2 - Organizar e financiar a oferta de serviços de saúde à população portuguesa (OPSS, 2007) ${ }^{(1)}$

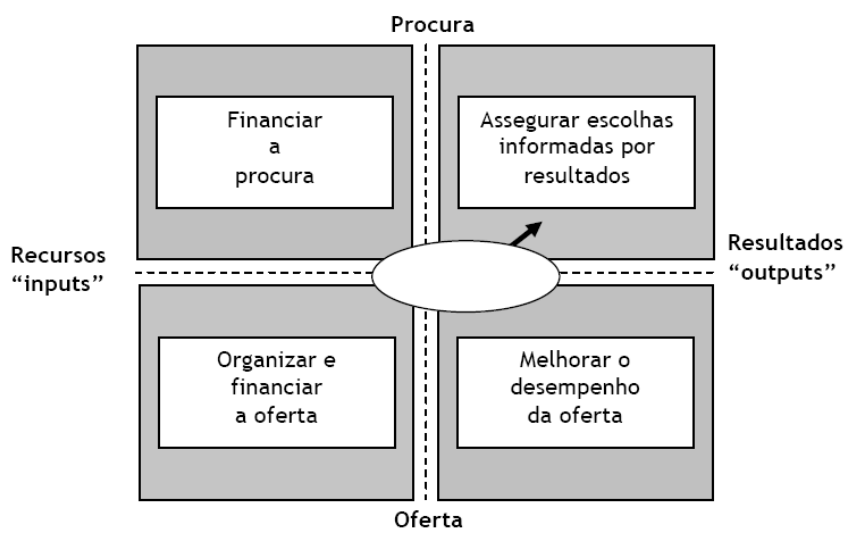

Figura 3 - Da lógica da "oferta de recursos" para lógica "da procura de resultados" (OPSS, 2007) $)^{(1)}$ 
dos serviços de saúde (quer em termos de instalações, quer na adopção de novas tecnologias) e na melhoria do acesso a recursos em saúde. Globalmente podemos dizer que se tem verificado um esforço na tentativa de melhorar a organização e gestão do sistema de saúde. Contudo, estas reformas bem-intencionadas foram, muitas vezes, implementadas de forma incompleta, quer por limitações de gestão, resistência à mudança ou descontinuidade das políticas de saúde (caracterizadas por alterações substanciais nas agendas políticas).

Podemos mesmo afirmar, que este esforço tem-se traduzido nos últimos tempos, numa procura constante em deslocar o enfoque da governação do sistema de saúde dos "recursos" para os "resultados", da "organização e financiamento da oferta" para a "melhoria do desempenho da oferta". Não nos podemos alhear das dificuldades com que o sistema de saúde português se tem defrontado, de eventual insustentabilidade financeira. Neste sentido, para a manutenção de um modelo social, solidário e universal, torna-se necessário o desenvolvimento de estratégias que conduzam ao abrandamento do ritmo de crescimento da despesa pública com a saúde e permitam a sustentabilidade financeira do sistema de saúde.

Torna-se, por isso, necesário centrar a atenção na "visão para o futuro da Saúde em Portugal”, nomeadamente no esforço que Portugal tem dedicado à "gestão da mudança do sistema de saúde português".

\section{O SISTEMA DE SAÚdE PORTUGUÊS: UMA VISÃO PARA O FUTURO DA SAÚdE EM PORTUGAL}

De forma a obter mais saúde para todos, o sistema de saúde português tem apostado a sua intervenção em duas estratégias: centrar as intervenções na família e no ciclo de vida e abordar os problemas de saúde através de uma aproximação à gestão da doença.

Dando continuidade ao Plano Nacional de Saúde 2004-2010, o Observatório do Quadro Comunitário de Apoio III (9), apresenta uma visão para o futuro da saúde em Portugal - denominada de "Saúde 2015", onde se reforça a necessidade de "um sistema de saúde que promova a iniciativa e a autonomia individual e os princípios da coesão social". Ou seja, um sistema de saúde onde tenham lugar iniciativas efectivas e justamente apoiadas, de promoção de saúde e que proporciona acesso a cuidados de saúde de qualidade, e que: facilite escolhas informadas que reflectem valores individuais e colectivos, esteja centrado num "novo serviço público" atento às pessoas e aos profissionais de saúde, assegure formas de articulação entre os sectores público, social e privado, compatíveis com os objectivos do sistema de saúde e com a qualidade dos instrumentos de governação disponíveis.

O Plano Nacional de Saúde 2004-2010(8), estabelece como uma das orientações estratégicas para a gestão da mudança "Reorientar o Sistema de Saúde", referindo que os investimentos previstos no sistema de saúde deverão contemplar ou providenciar ao cidadão um atendimento de qualidade, em tempo útil (melhoria do acesso), com efectividade, humanidade e custos sustentáveis ao longo do tempo.

Para tal, a mudança encontra-se focalizada, essencialmente, na Reforma Hospitalar (centrada no processo de empresarialização dos hospitais do SNS e na Rede Nacional de Cuidados Continuados Integrados) e na Reforma dos Cuidados de Saúde Primários, que integra a reconfiguração dos Centros de Saúde, a extinção das Regiões e a reestruturação das ARS.

\section{A Reforma hospitalar}

Assistimos a uma aposta no processo de empresarialização dos hospitais do SNS, numa gestão mais empresarial, com uma responsabilização clara dos gestores, apoiados por informação mais acessível e resultante de uma maior atenção ao sistema de informação; no desenvolvimento de parcerias com os sectores privado e social; numa maior coordenação vertical, entre níveis de cuidados, através de redes de referenciação e plataformas de articulação com outras forças vivas da comunidade.

O desenvolvimento da Rede Nacional de Cuidados Continuados Integrados ${ }^{(8,10)}$ é um processo evolutivo suportado em fases pré-definidas que se estendem até 2016. Esta Rede é constituída por diferentes tipologias de cuidados em conformidade com as diferentes necessidades das populações e das localidades. De entre as diferentes tipologias de internamento, destacam-se os conceitos inovadores materializados em:

- Unidades de internamento de Convalescença (internamento de curta duração);

- Unidades de internamento de Média-duração e reabilitação (internamento activo ou de reabilitação para a vida activa);

- Unidades de internamento de Longa-duração e permanente (internamento com actividades de vida diária);

- Unidades de internamento de Cuidados Paliativos (apoio durante o estado terminal da vida humana)

A Rede Nacional de Cuidados Continuados Integrados (RNCCI) prevê o apoio domiciliário a pessoas em situação de dependência, com necessidade de cuidados de saúde e apoio social, pelas equipas dos Centros de Saúde (CS) em articulação com as instituições da Rede Solidária. 
Neste contexto, e no domínio da RNCCI, a prestação dos cuidados de saúde e de apoio social, baseada num modelo de intervenção integrada e articulada, é assegurada por equipas domiciliárias, designadamente as equipas de saúde familiar das USF, de cuidados continuados integrados (ECCI) e a comunitária de suporte em cuidados paliativos (ECSCP), constituindo-se esta última, como um núcleo especializado das primeiras. Cada CS deverá, assim, incorporar na sua nova estrutura organizacional uma Unidade de Cuidados na Comunidade (UCC), cujo âmbito de actuação será a intervenção a grupos e comunidade, onde se integrarão as ECCI e ECSCP ${ }^{(11)}$.

\section{Reforma dos Cuidados de Saúde Primários}

Para a Missão para os Cuidados de Saúde Primários"(12), "a reforma dos cuidados de saúde primários, no âmbito da qual a reconfiguração dos centros de saúde é uma peça essencial, deve ser lida e compreendida no quadro mais vasto da reforma da administração pública que está em curso. Os princípios de racionalização de estruturas e recursos, de simplificação e de melhoria da qualidade...". Neste contexto, a agregação de recursos e estruturas de gestão, eliminando concorrências estruturais, permite a redução das despesas e viabiliza estratégias regionais ao nível dos cuidados de saúde primários que vão ao encontro das necessidades e expectativas da população.

Como peça vital no processo de Reforma dos Cuidados de Saúde Primários surge o redesenho organizacional de toda a estrutura da administração, regional e local, orientado à desconcentração na tomada de decisões, ao reforço dos mecanismos de contratualização e à implantação da gestão por objectivos. E este será, porventura, em termos de redesenho organizacional, um processo com uma dimensão sem precedentes na história do SNS.

Ao decidir-se pôr a tónica na criação das unidades de saúde familiar, como principal interface entre o cidadão e o seu sistema de saúde, na reforma da saúde fez-se uma escolha original e acertada para as pessoas que frequentam o SNS.

A criação das Unidades de Saúde Familiares*, na vertente assistencial directa, e a reorganização dos centros de saúde - com a criação de unidades de decisão gestionária mais próximas dos cidadãos e dos serviços - são dois aspectos cruciais de um conjunto de transformações que, ao nível da saúde em geral e dos CSP em particular, visam responder com maior satisfação e eficácia às necessidades das pessoas. Este processo está a ser desenvolvido em dois momentos:

- Criação das unidades de saúde familiar;

- Inserção das unidades periféricas nas novas agregações de centros de saúde (agrupamentos de centros de saúde), como organizações em rede, que substituem as sub-regiões de saúde.

Os Agrupamentos de Centros de Saúde (ACES) constituídos por várias unidades funcionais, que agrupam um ou mais centros de saúde, têm por missão garantir a prestação de cuidados de saúde primários à população de determinada área geográfica.

Os ACES (Figura 4) podem compreender as seguintes unidades funcionais:

- Unidade de saúde pública;
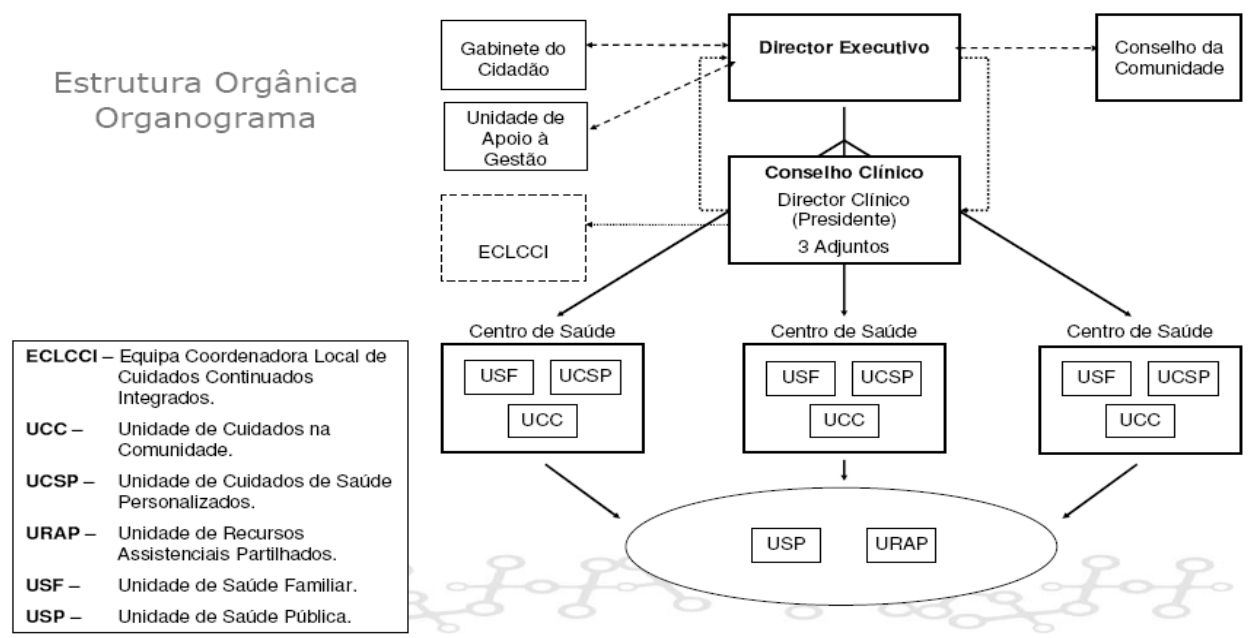

Figura 4- Estrutura orgânica dos agrupamentos de Centros de Saúde (Missão para os Cuidados de Saúde Primários - Ministério da Saúde, 2007) ${ }^{(13)}$

\footnotetext{
* As Unidades de Saúde Familiar (USF) são pequenas unidades operativas dos Centros de Saúde com autonomia funcional e técnica, que contratualizam objectivos de acessibilidade, adequação, efectividade, eficiência e qualidade, e que garantem aos cidadãos inscritos nessa unidade, um conjunto de cuidados de saúde.
} 
- Unidade de recursos assistenciais partilhados;

- Unidade de saúde familiar;

- Unidade de cuidados de saúde personalizados;

- Unidade de cuidados na comunidade.

As Unidades de Saúde Pública vão funcionar como observatório de saúde da população, permitindo uma detalhada caracterização da área geodemográfica e das necessidades de saúde da população sob sua dependência, em estreita e directa articulação com os serviços e instituições prestadoras de cuidados de saúde. A Unidade de Recursos Assistenciais Partilhados presta serviços de consultadoria e assistenciais às unidades funcionais e organiza ligações funcionais aos serviços hospitalares. Cada Unidade de Cuidados de Saúde Personalizados tem como missão garantir a prestação de cuidados de saúde personalizados à população inscrita de uma determinada área geográfica. A Unidade de Cuidados na Comunidade presta cuidados de saúde e apoio psicológico e social de âmbito domiciliário e comunitário, especialmente às pessoas, famílias e grupos mais vulneráveis, em situação de maior risco ou dependência física e funcional ou doença que requeira acompanhamento próximo, e actua ainda na educação para a saúde, na integração em redes de apoio à família e na implementação de unidades móveis de intervenção, garantindo a continuidade e qualidade dos cuidados prestados.

\section{CONSIDERAÇÕES FINAIS}

Um dos pontos centrais na Reforma da Saúde em Portugal registou-se em 1990, com a publicação da Lei de Bases da Saúde, que define o sistema de saúde português como uma estrutura que visa a efectivação do direito à protecção da saúde. A saúde passa a ser da responsabilidade não só do Estado mas também de cada indivíduo, e das iniciativas sociais e privadas. O sistema de saúde português passa a ser caracterizado pela coexistência de três sistemas articulados entre si: o Serviço Nacional de Saúde (abrangendo todas as instituições e serviços oficiais prestadores de cuidados de saúde dependentes do Ministério da Saúde); subsistemas de saúde públicos de apoio (entidades públicas que desenvolvem actividades de promoção, prevenção e tratamento na área da saúde); e, todas as entidades privadas e todos os profissionais livres que acordem com o SNS a prestação de cuidados ou de actividade de saúde. O sistema de saúde português, como o de outros países, tem-se desenvolvido na lógica da organização e financiamento da oferta de serviços de saúde à população do país. Este sistema constitui uma abordagem com uma forte base social de apoio no sistema político e nas profissões da saúde. De salientar que o Serviço Nacional de Saúde é definido, pelo menos no plano normativo, como sendo universal, geral e tendencialmente gratuito. No entanto, é cada vez mais evidente que esta aproximação não permite fazer face adequadamente aos importantes desafios de acesso, qualidade, efectividade e eficiência que os sistemas de saúde têm que enfrentar de uma forma cada vez mais crítica.

De qualquer das formas, independentemente do tipo de evolução do sistema de saúde português, não temos dúvidas que o serviço público de saúde desempenhará sempre um papel fundamental, devendo por isso apostar:

- na qualidade da interface com a comunidade (relação cidadão/profissionais de saúde, acesso do cidadão aos cuidados de saúde);

- num compromisso com resultados (antecipação e avaliação dos resultados: gestão clínica e gestão do desempenho);

- na liderança, na inovação (gerir a informação, conhecimento e comunicação);

- e, na missão de serviço público (ética, integração, qualidade).

Nunca nos deveremos esquecer que "os sistemas de saúde devem contribuir para a equidade e justiça social"»11).

\section{REFERÊNCIAS}

1. Observatório Português dos Sistemas de Saúde. Luzes e sombras: a governação em saúde. Coimbra: Centro de Estudos e Investigação em Saúde da Universidade de Coimbra (CEISUC); 2007. 226 p.

2. Missão para os Cuidados de Saúde Primários. Cuidados Continuados Integrados nos Cuidados de Saúde Primários: Carteira de Serviços. Ministério da Saúde, Lisboa [Internet]. 2007 [cited 2008 Set 25]. Available from: URL:http:// www.fnam.pt/images/ACS_MCSP.pdf

3. Portugal. Ministério da Saúde. Assembléia da República. Lei 56/79 de 15 de Setembro de 1979. Cria, no âmbito do Ministério dos Assuntos Sociais, o Serviço Nacional de Saúde (SNS), pelo qual o Estado assegura o direito à protecção da saúde, nos termos da Constituição. Diário da República, Lisboa, I Série A (15) Setembro, p. 2357.

4. Portugal. Ministério da Saúde. Assembléia da República. Lei n. ${ }^{\text {48/90, de }} 24$ de Agosto de 1990. Lei de Bases da Saúde. Diário da República, Lisboa, I Série A (195), p. 3452-9.

5. Portugal. Ministério da Saúde. Assembléia da República. Decreto-Lei 11/93. Estatuto do Serviço Nacional de Saúde. Diário da República, Lisboa, I Série A (12) 15 de Janeiro 1993, p. 122-34.

6. Portugal. Ministério da Saúde. Assembléia da República. Decreto-Lei 156/99, de 10 de Maio de 1999. Regime dos Sistemas Locais de Saúde. Diário da República, Lisboa, I Série A (108), p. 2421-4.

7. OCDE. OECD health data 2006 [CD-ROM]: statistics and indicators for 30 countries. Paris: OECD: IRDES; 2006. Version 06/26/2006.

8. Portugal. Ministério da Saúde. Plano Nacional de Saúde 2004/2010: mais saúde para todos. Orientações estratégicas. Lisboa: Direcção-Geral da Saúde; 2004. v.2 
9. Sakellarides C, Reis V, Escoval A, Conceição C, Barbosa P. O futuro do Sistema de Saúde Português. "Saúde 2015". Observatório do QCA III. Lisboa: Universidade Nova de Lisboa, Escola Nacional de Saúde Pública; 2006.

10. Portugal. Ministério da Saúde. Assembléia da República. Decreto-Lei no 101/2006, de 6 de Junho de 2006. Cria a Rede Nacional de Cuidados Continuados Integrados. Diário da República, I Série A (109), p. 3856-65.

11. World Health Organization. The world health report 2008: primary health care: now more than ever. [Geneva,
Switzerland] : World Health Organization, c2008.

12. Missão para os Cuidados de Saúde Primários, 2007. Proposta para a reconfiguração dos centros de saúde - Criação do Agrupamento de Centros de Saúde (ACS), Ministério da Saúde, Lisboa, [Internet]. 2007 [cited 2008 Set 25]. Available from: URL:http://www.fnam.pt/images/ ACS_MCSP.pdf

13. Pisco L. Reforma dos cuidados de saúde primários. Reconfiguração dos Centros de Saúde Criação de Agrupamentos de Centros de Saúde, Viseu 12 de Junho 2007 